\title{
On multivalent starlike functions
}

\author{
Mamoru Nunokawa, Janusz Sokół, Nikola
}

Tuneski and Biljana Jolevska-Tuneska

\begin{abstract}
We prove some new sufficient conditions for function to be $p$-valent, or $p$-valently starlike in the unit disc.
\end{abstract}

Mathematics Subject Classification (2010): 30C45, 30C80.

Keywords: Analytic functions, univalent functions, Ozaki's condition.

\section{Introduction}

Let $\mathcal{H}(\mathbb{D})$ denote the class of all analytic functions in the open unit disk

$$
\mathbb{D}=\{z \in \mathbb{C}:|z|<1\} .
$$

For $n$ a positive integer and $a \in \mathbb{C}$, let

$$
\mathcal{H}[a, n]=\left\{f \in \mathcal{H}(\mathbb{D}): f(z)=a+a_{n} z^{n}+a_{n+1} z^{n+1}+\cdots\right\} .
$$

Especially, let $\mathcal{A}_{p}$ be the subclass of $\mathcal{H}(\mathbb{D})$ consisting of the functions of the form

$$
f(z)=z^{p}+\sum_{n=1}^{\infty} a_{p+n} z^{p+n}
$$

and $\mathcal{A} \equiv \mathcal{A}_{1}$. For more details see $[1,4,8]$.

Further, a function $f \in \mathcal{A}_{p}, p=2,3, \ldots$, is said to be $p$-valently (or multivalently) starlike of order $\alpha, 0 \leq \alpha<p$, if

$$
\mathfrak{R e}\left\{\frac{z f^{\prime}(z)}{f(z)}\right\}>\alpha \quad(z \in \mathbb{D}) .
$$

The class of all such functions is usually denoted by $\mathcal{S}_{p}^{*}(\alpha)$. For $p=1$ we receive the well known class of normalized starlike univalent functions.

It is well known result from the theory of univalent functions due to Marx and Strohhacker $([3,10]))$ that when $f \in \mathcal{A}$,

$$
\mathfrak{R e}\left\{1+\frac{z f^{\prime \prime}(z)}{f^{\prime}(z)}\right\}>0 \quad(z \in \mathbb{D}) \quad \Rightarrow \quad \mathfrak{R e}\left\{\frac{z f^{\prime}(z)}{f(z)}\right\}>\frac{1}{2} \quad(z \in \mathbb{D}) .
$$


In this paper we study the question, that naturally rises, about the relation between the expressions $\frac{z f^{(p)}(z)}{f^{(p-1)}(z)}$ and $\frac{z f^{(p-1)}(z)}{f^{(p-2)}(z)}$, i.e., between $\frac{z f^{(p)}(z)}{f^{(p-1)}(z)}$ and $\frac{z f^{\prime}(z)}{f(z)}$. We close the paper with a sharp (necessary and sufficient) condition when the following implication holds:

$$
\mathfrak{R e}\left\{\frac{z f^{(p)}(z)}{f^{(p-1)}(z)}\right\}>\alpha \quad(z \in \mathbb{D}) \quad \Rightarrow \quad \mathfrak{R e}\left\{\frac{z f^{\prime}(z)}{f(z)}\right\}>\beta \quad(z \in \mathbb{D}),
$$

for the case $\frac{p-1}{2} \leq \alpha<p$. We also give non-sharp result for $0 \leq \alpha<\frac{p-1}{2}$ and the sharp version in this case remains an open problem.

For the study we use two different methods, one based on a generalized Jack lemma, and other, based on a result from the theory of differential subordinations. The first method will bring stronger conclusion, but that conclusion will hold for smaller class than the corresponding conclusion received by the second method, which will even be sharp for $\frac{p-1}{2} \leq \alpha<p$. Further in the paper, this will be discussed again, in more details.

\section{Results based on generalized Jack lemma}

The famous Jack lemma was originally published in [2] and its generalization will be used for proving the main result in this section.

First, let denote by $\mathcal{Q}$ the class of functions $f$ that are analytic and injective on $\overline{\mathbb{D}} \backslash E(f)$, where

and are such that

$$
E(f):=\left\{\zeta: \zeta \in \partial \mathbb{D} \text { and } \lim _{z \rightarrow \zeta} f(z)=\infty\right\}
$$

$$
f^{\prime}(\zeta) \neq 0 \quad(\zeta \in \partial(\mathbb{D}) \backslash E(f))
$$

Now, the generalization of the Jack lemma that we will need is the following

Lemma 2.1 ([4]). Let $q \in \mathcal{Q}$ with $q(0)=a$ and let

$$
p(z)=a+a_{n} z^{n}+\cdots
$$

be analytic in $\mathbb{D}$ with

$$
p(z) \not \equiv a \text { and } n \in \mathbb{N}=\{1,2,3, \cdots\} .
$$

If $p$ is not subordinate to $q$, then there exist points

$$
z_{0}=r_{0} e^{i \theta} \in \mathbb{D} \text { and } \zeta_{0} \in \partial \mathbb{D} \backslash E(q),
$$

for which

$$
\begin{gathered}
p\left(|z|<r_{0}\right) \subset q(\mathbb{D}), \\
p\left(z_{0}\right)=q\left(\zeta_{0}\right)
\end{gathered}
$$

and

$$
z_{0} p^{\prime}\left(z_{0}\right)=k \zeta_{0} q^{\prime}\left(\zeta_{0}\right)
$$

for some $k \geq n$.

The next lemma will also be needed. 
Lemma $2.2([7])$. Let $p(z), p(0)=1$, be analytic in $|z|<1$ and suppose that there exists a point $z_{0},\left|z_{0}\right|<1$ such that

$$
\mathfrak{R e}\{p(z)\}>\beta \text { for }|z|<\left|z_{0}\right|
$$

and

$$
\mathfrak{R e}\left\{p\left(z_{0}\right)\right\}=\beta,
$$

where $0 \leq \beta<1$ and $p\left(z_{0}\right) \neq \beta$. Then we have the following properties:

(1) For the case $\arg \left\{p\left(z_{0}\right)\right\}>0$,

$$
\begin{aligned}
\frac{z_{0} p^{\prime}\left(z_{0}\right)}{p\left(z_{0}\right)-\beta} & =\left\{\frac{-i}{|p(z)-\beta|} \frac{\mathrm{d}|p(z)-\beta|}{\mathrm{d} \theta}\right\}_{z=z_{0}} \\
& =i k
\end{aligned}
$$

where $k$ is a real number and $k \geq\left(a^{2}+1\right) /(2 a), p\left(z_{0}\right)-\beta=i$ and $a>0$.

(2) For the case $\arg \left\{p\left(z_{0}\right)\right\}<0$,

$$
\begin{aligned}
\frac{z_{0} p^{\prime}\left(z_{0}\right)}{p\left(z_{0}\right)-\beta} & =\left\{\frac{-i}{|p(z)-\beta|} \frac{\mathrm{d}|p(z)-\beta|}{\mathrm{d} \theta}\right\}_{z=z_{0}} \\
& =-i k
\end{aligned}
$$

where $k$ is a real number and $k \geq\left(a^{2}+1\right) /(2 a), p\left(z_{0}\right)-\beta=-i$ and $a>0$.

Before giving the main result of this section let prove the following lemma that we will also need.

Lemma 2.3. Let $p(z), p(0)=1$, be analytic in the unit disk $\mathbb{D}$ and suppose that there exists a point $z_{0},\left|z_{0}\right|<1$, such that

$$
\mathfrak{R e}\{p(z)\}>\beta \text { for }|z|<\left|z_{0}\right|
$$

and

$$
\mathfrak{R e}\left\{p\left(z_{0}\right)\right\}=\beta, \quad a=\left|\mathfrak{I m}\left\{p\left(z_{0}\right)\right\}\right|>0,
$$

where $0 \leq \beta<1$. Then, there are the real numbers $k$ and $m$ such that

$$
k \geq \frac{a^{2}+1}{2 a}, \quad m \geq 1
$$

and

$$
z_{0} p^{\prime}\left(z_{0}\right)=\left\{\begin{array}{lll}
-k a & \text { when } & p\left(z_{0}\right) \neq \beta \\
-m(1-\beta) / 2 & \text { when } & p\left(z_{0}\right)=\beta .
\end{array}\right.
$$

Proof. For the case $p\left(z_{0}\right) \neq \beta, 0<\beta<1$, we may apply Lemma 2.2. If $\arg \left\{p\left(z_{0}\right)\right\}>0$, then from (2.2) we have

$$
\frac{z_{0} p^{\prime}\left(z_{0}\right)}{p\left(z_{0}\right)-\beta}=\frac{z_{0} p^{\prime}\left(z_{0}\right)}{i a}=i k,
$$

hence $z_{0} p^{\prime}\left(z_{0}\right)=-k a$. For the case $\arg \left\{p\left(z_{0}\right)\right\}<0$, from (2.3) we have

$$
\frac{z_{0} p^{\prime}\left(z_{0}\right)}{p\left(z_{0}\right)-\beta}=\frac{z_{0} p^{\prime}\left(z_{0}\right)}{-i a}=-i k,
$$

hence $z_{0} p^{\prime}\left(z_{0}\right)=-k a$. 
For the case $p\left(z_{0}\right)=\beta, 0 \leq \beta<1$, we will apply Lemma 2.1. If $p\left(z_{0}\right)=\beta$, then

$$
p(z) \nprec \frac{1+(1-2 \beta) z}{1-z}:=g_{\beta}(z),
$$

moreover

$$
p\left(z_{0}\right)=\beta=g_{\beta}\left(\zeta_{0}\right), \zeta_{0}=-1 .
$$

From this and from (2.4), by Lemma 2.1, it follows that

$$
z_{0} p^{\prime}\left(z_{0}\right)=m \zeta_{0} g_{\beta}^{\prime}\left(\zeta_{0}\right), \zeta_{0}=-1,
$$

for some $m \geq 1$. For the function $g_{\beta}$ we have

$$
g_{\beta}^{\prime}(z)=\left.\frac{2(1-\beta)}{(1-z)^{2}}\right|_{z=\zeta_{0}}=\frac{1-\beta}{2} .
$$

Applying this in (2.6) we obtain the second case in (2.5).

Finally, we can formulate and prove the main result of this section.

Theorem 2.4. Let $f \in \mathcal{A}_{p}, p \geq 2$, be analytic in $\mathbb{D}$. Assume that

$$
\mathfrak{R e}\left\{\frac{z f^{(p)}(z)}{f^{(p-1)}(z)}\right\}>0 \quad(z \in \mathbb{D})
$$

and

$$
\frac{z f^{(p-1)}(z)}{2 f^{(p-2)}(z)} \neq \frac{2}{3} \quad(z \in \mathbb{D}) .
$$

Then,

$$
\mathfrak{R e}\left\{\frac{z f^{(p-1)}(z)}{f^{(p-2)}(z)}\right\}>\frac{4}{3} \quad(z \in \mathbb{D}) .
$$

Proof. Let us put

$$
p(z)=\frac{z f^{(p-1)}(z)}{2 f^{(p-2)}(z)},
$$

such that $p(0)=1$. It follows that

$$
\frac{z f^{(p)}(z)}{f^{(p-1)}(z)}=2 p(z)+\frac{z p^{\prime}(z)}{p(z)}-1 .
$$

If there exists a point $z_{0} \in \mathbb{D}$ such that

$$
\mathfrak{R e}\left\{p\left(z_{0}\right)\right\}>\frac{2}{3} \text { for }|z|<\left|z_{0}\right|
$$

and

then, from Lemma 2.2, we have

$$
\mathfrak{R e}\left\{p\left(z_{0}\right)\right\}=\frac{2}{3},
$$

$$
\frac{z_{0} p^{\prime}\left(z_{0}\right)}{p\left(z_{0}\right)-2 / 3}=i k, \quad k \geq 1,
$$


where $k \geq\left(a^{2}+1\right) /(2 a)$ if $p\left(z_{0}\right)=2 / 3+i a$ and $a>0$, while $k \leq-\left(a^{2}+1\right) /(2 a)$ if $p\left(z_{0}\right)=2 / 3-i a$ and $a>0$. For the case $p\left(z_{0}\right)=2 / 3+i a$ and $a>0$, from (2.10) and (2.11), we have

$$
\begin{aligned}
\frac{z_{0} f^{(p)}\left(z_{0}\right)}{f^{(p-1)}\left(z_{0}\right)} & =2 p\left(z_{0}\right)+\frac{z_{0} p^{\prime}\left(z_{0}\right)}{p\left(z_{0}\right)}-1 \\
& =2 p\left(z_{0}\right)+\frac{z_{0} p^{\prime}\left(z_{0}\right)}{p\left(z_{0}\right)-2 / 3} \frac{p\left(z_{0}\right)-2 / 3}{p\left(z_{0}\right)}-1 \\
& =\frac{4}{3}+2 i a+i k \frac{i a}{2 / 3+i a}-1 \\
& =\frac{1}{3}-\frac{a k}{2 / 3+i a} .
\end{aligned}
$$

Hence, for $a>0$, we obtain

$$
\begin{aligned}
\mathfrak{R e}\left\{\frac{z_{0} f^{(p)}\left(z_{0}\right)}{f^{(p-1)}\left(z_{0}\right)}\right\} & =\mathfrak{R e}\left\{\frac{1}{3}-\frac{a k}{2 / 3+i a}\right\} \\
& =\frac{1}{3}-\frac{2 a k / 3}{4 / 9+a^{2}} \\
& \leq \frac{1}{3}-\frac{6 a \frac{a^{2}+1}{2 a}}{4+9 a^{2}} \\
& =\frac{1}{3}-\frac{3 a^{2}+3}{4+9 a^{2}} \\
& =\frac{4-9}{3\left(4+9 a^{2}\right)} \\
& <0 .
\end{aligned}
$$

This contradicts with (2.7). For the case $p\left(z_{0}\right)=2 / 3-i a, k \leq-\left(a^{2}+1\right) /(2 a)$ and $a>0$, from (2.11) and (2.12), we have

$$
\begin{aligned}
\mathfrak{R e}\left\{\frac{z_{0} f^{(p)}\left(z_{0}\right)}{f(p-1)\left(z_{0}\right)}\right\} & =\mathfrak{R e}\left\{\frac{4}{3}-2 i a+i k \frac{-i a}{2 / 3-i a}-1\right\} \\
& =\mathfrak{R e}\left\{\frac{1}{3}+\frac{a k}{2 / 3-i a}\right\} \\
& =\frac{1}{3}+\frac{2 a k / 3}{4 / 9+a^{2}} \\
& \leq \frac{1}{3}-\frac{6 a \frac{a^{2}+1}{2 a}}{4+9 a^{2}} \\
& =\frac{1}{3}-\frac{3 a^{2}+3}{4+9 a^{2}} \\
& =\frac{4-9}{3\left(4+9 a^{2}\right)} \\
& <0 .
\end{aligned}
$$


This also contradicts with (2.7). Therefore,

$$
\mathfrak{R e}\{p(z)\}>\frac{2}{3} \quad(z \in \mathbb{D})
$$

which is equivalent to $(2.9)$.

The condition (2.8) in the above theorem is necessary in order to apply Lemma 2.2 , i.e., without $(2.8)$ we cannot obtain $4 / 3$ in $(2.9)$.

\section{Results based on differential subordinations}

In this section we will give sharp, i.e., necessary and sufficient, conditions when the following implications hold.

$$
\begin{array}{ll}
\mathfrak{R e}\left\{\frac{z f^{(p)}(z)}{f^{(p-1)}(z)}\right\}>\alpha \quad(z \in \mathbb{D}) & \Rightarrow \quad \mathfrak{R e}\left\{\frac{z f^{(p-1)}(z)}{f^{(p-2)}(z)}\right\}>\beta \quad(z \in \mathbb{D}) \\
\mathfrak{R e}\left\{\frac{z f^{(p)}(z)}{f^{(p-1)}(z)}\right\}>\alpha \quad(z \in \mathbb{D}) & \Rightarrow \quad \mathfrak{R e}\left\{\frac{z f^{\prime}(z)}{f(z)}\right\}>\beta \quad(z \in \mathbb{D})
\end{array}
$$

For proving the main result we will use the following lemma from the theory of differential subordinations.

Lemma 3.1 (Theorem 2.3i(i), p. 35, [4]). Let $\Omega \subset \mathbb{C}$ and suppose that the function $\psi: \mathbb{C}^{2} \times \mathbb{D} \rightarrow \mathbb{C}$ satisfies $\psi(i x, y ; z) \notin \Omega$ for all $x \in \mathbb{R}, y \leq-n\left(1+x^{2}\right) / 2$, and $z \in \mathbb{D}$. If $q \in H[1, n]$ and $\psi\left(q(z), z q^{\prime}(z) ; z\right) \in \Omega$ for all $z \in \mathbb{D}$, then $\mathfrak{R e}\{q(z)\}>0, z \in \mathbb{D}$.

Theorem 3.2. Let $p$ be a positive integer, $p \geq 3$ and $\beta_{1} \leq \beta<2$, where $\beta_{1}=\frac{1+\sqrt{17}}{4}=$ $1.280776 \ldots$... Also, let

$$
\alpha \equiv \alpha(\beta)=-\frac{1}{2}+\beta-\frac{1}{\beta}
$$

If $f \in \mathcal{A}_{p}$ and

$$
\mathfrak{R e}\left\{\frac{z f^{(p)}(z)}{f^{(p-1)}(z)}\right\}>\alpha \quad(z \in \mathbb{D}),
$$

then

$$
\mathfrak{R e}\left\{\frac{z f^{(p-1)}(z)}{f^{(p-2)}(z)}\right\}>\beta \quad(z \in \mathbb{D}) .
$$

This result is sharp, i.e. $\alpha(\beta)$ can not be replaced by a smaller number so that the implication to remains true.

Proof. In the view of Lemma 3.1, let define the functions

$$
q(z)=\frac{1}{2-\beta}\left\{\frac{z f^{(p-1)}(z)}{f^{(p-2)}(z)}-\beta\right\} \quad(z \in \mathbb{D})
$$

and

$$
\psi(r, s ; z)=\frac{s(2-\beta)}{r(2-\beta)+\beta}+r(2-\beta)+\beta-1,
$$


such that $q(z) \in \mathcal{H}[1,1]$ and

$$
\psi\left(q(z), z q^{\prime}(z) ; z\right)=\frac{z f^{(p)}(z)}{f^{(p-1)}(z)}
$$

Now, let consider

$$
\psi(i x, y ; z)=\frac{(2-\beta) y}{i x(2-\beta)+\beta}+i x(2-\beta)+\beta-1,
$$

where $x$ is real number, $y \leq-\left(1+x^{2}\right) / 2$ and $z \in \mathbb{D}$. We have

$$
\begin{aligned}
\mathfrak{R e}\{\psi(i x, y ; z)\} & =\beta-1+\frac{(2-\beta) \beta y}{(2-\beta)^{2} x^{2}+\beta^{2}} \\
& \leq \beta-1-\frac{(2-\beta) \beta \frac{1+x^{2}}{2}}{(2-\beta)^{2} x^{2}+\beta^{2}} \equiv \varphi(\beta, x)
\end{aligned}
$$

and

$$
\frac{\partial}{\partial x} \varphi(\beta, x)=\frac{4 \beta(1-\beta)(2-\beta) x}{\left[(2-\beta)^{2} x^{2}+\beta^{2}\right]^{2}} .
$$

Since $1<\beta_{1} \leq \beta<2$, we obtain that $\varphi(\beta, x)$ is a decreasing function of the variable $x$. So,

$$
\varphi(\beta, x) \leq \varphi(\beta, 0)=-\frac{1}{2}+\beta-\frac{1}{\beta}=\alpha(\beta)
$$

and from Lemma 3.1 we conclude that

$$
\mathfrak{R e}\left\{\frac{z f^{(p)}(z)}{f^{(p-1)}(z)}\right\}>\alpha(\beta) \quad(z \in \mathbb{D}) \quad \Rightarrow \quad \mathfrak{R e}\{q(z)\}>0 \quad(z \in \mathbb{D}),
$$

i.e.

$$
\mathfrak{R e}\left\{\frac{z f^{(p-1)}(z)}{f^{(p-2)}(z)}\right\}>\beta \quad(z \in \mathbb{D}) .
$$

Now, we will show that the result is sharp using the extremal function $f_{*}(z)$ defined by

$$
f_{*}^{(p-2)}(z)=\frac{p !}{2} z^{2} \cdot(1-z)^{-2(2-\beta)},
$$

(all powers are taken by their principal values) such that

$$
\frac{z f_{*}^{(p-1)}(z)}{f_{*}^{(p-2)}(z)}-\beta=\frac{1+z}{1-z} \cdot(2-\beta) \equiv q_{*}(z) .
$$

First, let note that for $z=0, \frac{z f_{*}^{(p-1)}(z)}{f_{*}^{(p-2)}(z)}=2$ and that $f_{*}(z)$ is analytic in $\mathbb{D}$, i.e. that $f_{*}(z) \in \mathcal{A}_{p}$. Further, recall that $\frac{1+z}{1-z}$ maps the unit disk onto the right half of the complex plane and the unit circle onto the imaginary axis. So, $\frac{z f_{*}^{(p-1)}(z)}{f_{*}^{(p-2)}(z)}$ maps the unit disk onto $\{\omega: \mathfrak{R e}\{\omega\}>\beta\}$.

In order to prove the sharpness of the result it is enough to show that the boundary of the image of the unit disk by the function $\frac{z f_{*}^{(p)}(z)}{f_{*}^{(p-1)}(z)}$ touches the vertical line through $\alpha(\beta)$. Otherwise, it would be possible to find $\alpha$ larger than $\alpha(\beta)$ that 
implies the conclusion (3.2). In that direction, let note that for any $|z|=1, z \neq 1$, there exists real number $x$ such that

$$
\begin{gathered}
q_{*}(z)=\frac{1+z}{1-z} \cdot(2-\beta)=i x(2-\beta) \\
z q_{*}^{\prime}(z)=\frac{2 z(2-\beta)}{(1-z)^{2}}=\frac{1}{2}\left\{\left(\frac{1+z}{1-z}\right)^{2}-1\right\}(2-\beta)=-\frac{1}{2}\left(x^{2}+1\right)(2-\beta)
\end{gathered}
$$

and

$$
\begin{aligned}
& \mathfrak{R e}\left\{\frac{z f_{*}^{(p)}(z)}{f_{*}^{(p-1)}(z)}\right\} \\
= & \mathfrak{R e}\left\{\frac{z q_{*}^{\prime}(z)}{q_{*}(z)+\beta}+q_{*}(z)+\beta-1\right\} \\
= & \mathfrak{R e}\left\{\frac{-\frac{1}{2}\left(x^{2}+1\right)(2-\beta)}{i x(2-\beta)+\beta}+i x(2-\beta)+\beta-1\right\} \\
= & \beta-1-\frac{(2-\beta) \beta \frac{1+x^{2}}{2}}{(2-\beta)^{2} x^{2}+\beta^{2}}=\varphi(\beta, x) .
\end{aligned}
$$

Therefore, for $z=-1$ we have

$$
\mathfrak{R e}\left\{\frac{z f_{*}^{(p)}(z)}{f_{*}^{(p-1)}(z)}\right\}=\varphi(\beta, 0)=\beta-1-\frac{2-\beta}{2 \beta}=\alpha(\beta) .
$$

So, $\frac{z f_{*}^{(p)}(z)}{f_{*}^{(p-1)}(z)}$ maps the unit disk onto a region that touches, from the right hand side, the vertical line through $\alpha(\beta)$. This completes the proof of the sharpness of the result.

Remark 3.3. It can be verified that the function $\varphi(\beta, x)$ is negative if, and only if, $0 \leq \beta<\beta_{1}$ and this case is not of interest because it leads to negative values of $\alpha$.

Theorem 3.2 can be rewritten in the following, equivalent form.

Theorem 3.4. Let $p$ be a positive integer such that $p \geq 3$. Also, let $0 \leq \alpha<1$ and

$$
\beta \equiv \beta(\alpha)=\frac{1+2 \alpha+\sqrt{(1+2 \alpha)^{2}+16}}{4} .
$$

If $f \in \mathcal{A}_{p}$ and

$$
\mathfrak{R e}\left\{\frac{z f^{(p)}(z)}{f^{(p-1)}(z)}\right\}>\alpha \quad(z \in \mathbb{D})
$$

then

$$
\mathfrak{R e}\left\{\frac{z f^{(p-1)}(z)}{f^{(p-2)}(z)}\right\}>\beta \quad(z \in \mathbb{D}) .
$$

This result is sharp, i.e. $\beta(\alpha)$ can not be replaced by a larger number so that the implication remains true. 
Proof. For the function $\alpha(\beta)$ defined by (3.1) we have $\frac{\partial}{\partial \beta} \alpha(\beta)=1+\frac{1}{\beta^{2}}>0$. So, $\alpha(\beta)$ is a strictly monotone function on the interval $\left[\beta_{1}, 2\right)$ and there exist its inverse function. It is not difficult to check that $\beta(\alpha)$ is that inverse function.

Remark 3.5. For $\alpha=0$ in the previous theorem we receive $\beta=\frac{1+\sqrt{17}}{4}=1.280776 \ldots$ which is smaller that $4 / 3$, received in Theorem 2.4. This does not mean that the result from Theorem 2.4 is stronger because it is obtained under the additional condition $\frac{z f^{(p-1)}(z)}{2 f^{(p-2)}(z)} \neq \frac{2}{3}$ for all $z \in \mathbb{D}$.

For simplicity of the proofs of the next corollaries we will reformulate Theorem 3.4 again.

Theorem 3.6. Let $l$ be a positive integer such that $l \geq 3$. Also, let $0 \leq \alpha<1$ and $\beta \equiv \beta(\alpha)$ be defined as in (3.3). If $g \in \mathcal{A}_{l}$ and

$$
\mathfrak{R e}\left\{\frac{z g^{(l)}(z)}{g^{(l-1)}(z)}\right\}>\alpha \quad(z \in \mathbb{D}),
$$

then

$$
\mathfrak{R e}\left\{\frac{z g^{(l-1)}(z)}{g^{(l-2)}(z)}\right\}>\beta \quad(z \in \mathbb{D}) .
$$

This result is sharp, i.e. $\beta(\alpha)$ can not be replaced by a larger number so that the implication remains true.

Now, as a corollary we obtain sharp information about the real part of $\frac{z f^{(k)}(z)}{f^{(k-1)}(z)}$ for any $2 \leq k \leq p-1$, having information about the real part of $\frac{z f^{(p)}(z)}{f^{(p-1)}(z)}$.

Corollary 3.7. Let $p$ and $k$ be positive integers such that $p \geq 3$ and $2 \leq k \leq p-1$. Also, let $0 \leq \alpha<1$ and let $\alpha_{i}, i=1,2, \ldots$, be a sequence defined by:

$$
\alpha_{1}=\beta(\alpha), \quad \alpha_{i}=\beta\left(\alpha_{i-1}-1\right),
$$

where $\beta(\alpha)$ is defined as in (3.3). If $f \in \mathcal{A}_{p}$ and

$$
\mathfrak{R e}\left\{\frac{z f^{(p)}(z)}{f^{(p-1)}(z)}\right\}>\alpha \quad(z \in \mathbb{D}),
$$

then

$$
\mathfrak{R e}\left\{\frac{z f^{(k)}(z)}{f^{(k-1)}(z)}-1\right\}>\alpha_{p-k} \quad(z \in \mathbb{D}) .
$$

This result is sharp, i.e., $\alpha_{p-k}$ can not be replaced by a larger number so that implication remains true.

Proof. Applying Theorem 3.6 with $g(z)=f(z)$ and $l=p$ we receive

$$
\mathfrak{R e}\left\{\frac{z f^{(p)}(z)}{f^{(p-1)}(z)}\right\}>\alpha \quad(z \in \mathbb{D}) \quad \Rightarrow \quad \mathfrak{R e}\left\{\frac{z f^{(p-1)}(z)}{f^{(p-2)}(z)}\right\}>\beta(\alpha)=\alpha_{1} \quad(z \in \mathbb{D}) .
$$


Further, for the function $g(z) \in \mathcal{A}_{p-1}$ defined by $g^{(p-2)}(z)=\frac{2}{p} \cdot \frac{f^{(p-2)}(z)}{z}$ and $l=p-1$ we have

$$
\mathfrak{R e}\left\{\frac{z g^{(l)}(z)}{g^{(l-1)}(z)}\right\}=\mathfrak{R e}\left\{\frac{z f^{(p-1)}(z)}{f^{(p-2)}(z)}-1\right\}>\beta(\alpha)-1 \quad(z \in \mathbb{D})
$$

and from Theorem 3.6 we obtain

$$
\mathfrak{R e}\left\{\frac{z f^{(p-2)}(z)}{f^{(p-3)}(z)}-1\right\}=\mathfrak{R e}\left\{\frac{z g^{(l-1)}(z)}{g^{(l-2)}(z)}\right\}>\beta(\beta(\alpha)-1)=\alpha_{2} \quad(z \in \mathbb{D}) .
$$

Applying Theorem 3.6 recursively ( $p-k$ times in total) we reach (3.4). The sharpness of the implication follows from the sharpness of Theorem 3.6.

\section{Remark 3.8.}

(i) The sequence defined in Corollary 3.7 is increasing and bounded between $\beta_{1}$ and 2. Thus, the recursive application of Theorem 3.6 is possible.

(ii) For $\alpha=0$ in Corollary 3.7 we receive an improvement of a result from [6] where the implication is proven with 0 on the place of $\alpha_{p-k}$ in (3.4).

For obtaining partially sharp information about the real part of $\frac{z f^{\prime}(z)}{f(z)}$, having information about the real part of $\frac{z f^{(p)}(z)}{f^{(p-1)}(z)}$, we need the following two results.

Lemma 3.9 ([9]). Let $p$ be a positive integer and $\frac{p-1}{2} \leq \alpha<p$. Also, let

$$
\beta \equiv \beta_{1}(\alpha, p)=\frac{p}{{ }_{2} F_{1}(1,2(p-\alpha) ; p+1 ; 1 / 2)} .
$$

If $f \in \mathcal{A}_{p}$ and

$$
\mathfrak{R e}\left\{1+\frac{z f^{\prime \prime}(z)}{f^{\prime}(z)}\right\}>\alpha \quad(z \in \mathbb{D})
$$

then

$$
\mathfrak{R e}\left\{\frac{z f^{\prime}(z)}{f(z)}\right\}>\beta \quad(z \in \mathbb{D}) .
$$

So, $\mathcal{K}_{p}(\alpha) \subset \mathcal{S}_{p}^{*}\left(\beta_{1}(\alpha, p)\right)$, i.e. $p$-valently convex functions of order $\alpha$, have $\beta_{1}(\alpha, p)$ order of p-valently starlikeness. This result is sharp, i.e., $\beta$ can not be replaced by $a$ number bigger than $\beta_{1}(\alpha, p)$ so that the implication remains true.

Lemma $3.10([5])$. Let $p$ be a positive integer and $0 \leq \alpha<p$. Also, let

$$
\beta \equiv \beta_{2}(\alpha, p)=\left\{\begin{array}{cl}
\frac{2(\alpha+p)-1+\sqrt{[2(\alpha+p)-1]^{2}-16 \alpha p}}{4} & \text { if } 0 \leq \alpha \leq \frac{p-1}{2} \\
\frac{2 \alpha-1+\sqrt{(2 \alpha-1)^{2}+8 p}}{4} & \text { if } \quad \frac{p-1}{2}<\alpha<p
\end{array} .\right.
$$

If $f \in \mathcal{A}_{p}$ and

then

$$
\mathfrak{R e}\left\{1+\frac{z f^{\prime \prime}(z)}{f^{\prime}(z)}\right\}>\alpha \quad(z \in \mathbb{D})
$$

$$
\mathfrak{R e}\left\{\frac{z f^{\prime}(z)}{f(z)}\right\}>\beta \quad(z \in \mathbb{D}) \text {. }
$$


So, $\mathcal{K}_{p}(\alpha) \subset \mathcal{S}_{p}^{*}\left(\beta_{2}(\alpha, p)\right)$, i.e. $p$-valently convex functions of order $\alpha$, have $\beta_{2}(\alpha, p)$ order of p-valently starlikeness.

Finally, here is a sharp solution of the general question studied in this paper.

Corollary 3.11. Let $p$ be a positive integer such that $p \geq 3$. Also, let $0 \leq \alpha<1, \alpha_{p-2}$ is defined as in Corollary 3.7 and

$$
\beta \equiv\left\{\begin{array}{l}
\beta_{2}\left(\alpha_{p-2}+2, p\right), \quad 0 \leq \alpha<\frac{p-1}{2} \\
\beta_{1}\left(\alpha_{p-2}+2, p\right), \quad \frac{p-1}{2} \leq \alpha<p
\end{array},\right.
$$

where functions $\beta_{1}(\alpha, p)$ and $\beta_{2}(\alpha, p)$ are defined as in Lemma 3.9 and Lemma 3.10. If $f \in \mathcal{A}_{p}$ and

then

$$
\mathfrak{R e}\left\{\frac{z f^{(p)}(z)}{f^{(p-1)}(z)}\right\}>\alpha \quad(z \in \mathbb{D}),
$$

$$
\mathfrak{R e}\left\{\frac{z f^{\prime}(z)}{f(z)}\right\}>\beta \quad(z \in \mathbb{D}) .
$$

For $\frac{p-1}{2} \leq \alpha<p$ the result is sharp.

Proof. For $k=2$ in Corollary 3.7 we receive that

$$
\mathfrak{R e}\left\{1+\frac{z f^{\prime \prime}(z)}{f^{\prime}(z)}\right\}>\alpha_{p-2}+2 \quad(z \in \mathbb{D}) .
$$

The rest follows from Lemma 3.9 and Lemma 3.10.

\section{References}

[1] Duren, P.L., Univalent Functions, Springer-Verlag, 1983.

[2] Jack, I.S., Functions starlike and convex of order $\alpha$, J. Lond. Math. Soc., 3(1971), 469474.

[3] Marx, A., Untersuchungen über schlichte Abbildungen, Math. Ann., 107(1932/33), 40-65.

[4] Miller, S.S., Mocanu, P.T., Differential Subordinations: Theory and Applications, Series of Monographs and Textbooks in Pure and Applied Mathematics, Vol. 225, Marcel Dekker Inc., New York/Basel, 2000.

[5] Nunokawa, M., Srivastava, H.M., Tuneski, N., Jolevska-Tuneska, B., Some MarxStrohhacker type results for a class of multivalent functions, Miskolc Math. Notes, 18(2017), no. 1, 353-364.

[6] Nunokawa, M., On the theory of multivalent functions, Tsukuba J. Math., 11(1987), no. 2, 273-286.

[7] Nunokawa, M., Sokól, J., Cho, N.E., Some applications of Jack's lemma, preprint.

[8] Owa, S., Srivastava, H.M., Current Topics in Analytic Function Theory, World Sci. Publ., River Edge, NJ, 1992.

[9] Srivastava, H.M., Patel, J., Mohapatra, G.P., A certain class of p-valently analytic functions, Math. Comput. Model., 41(2005), no. 2-3, 321-334.

[10] Strohhacker, E., Beitrage zür Theorie der schlichten Funktionen, Math. Z., 37(1933), 356-380. 
Mamoru Nunokawa

University of Gunma, Hoshikuki-cho 798-8

Chuou-Ward, Chiba, 260-0808, Japan

e-mail: mamoru-nuno@doctor.nifty.jp

Janusz Sokół

University of Rzeszów

Faculty of Mathematics and Natural Sciences

ul. Prof. Pigonia 1, 35-310 Rzeszów, Poland

e-mail: jsokol@prz.edu.pl

Nikola Tuneski

Faculty of Mechanical Engineering

Ss. Cyril and Methodius University in Skopje

Karpoš II b.b., 1000 Skopje, Republic of Macedonia

e-mail: nikola.tuneski@mf .edu.mk

Biljana Jolevska-Tuneska

Faculty of Electrical Engineering and Informational Technologies

Ss. Cyril and Methodius University in Skopje

Karpoš II b.b., 1000 Skopje, Republic of Macedonia

e-mail: biljanaj@feit.ukim.edu.mk 